\title{
Frequency of autoantibodies in patients with chronic periodontitis
}

\begin{abstract}
Background/objective: There has been an increasing interest in the relationship between periodontitis and systemic health. Several studies have suggested an association between periodontitis and systemic autoimmune diseases, such as rheumatoid arthritis. This study was conducted to compare the frequency of auto antibodies in chronic periodontitis patients and systemically healthy controls.
\end{abstract}

Materials and methods: This cross sectional study was carried out in the Department of Pathology, Allied Hospital Faisalabad, Pakistan. Blood samples of 90 subjects (45 with chronic periodontitis and 45 healthy controls) were taken by random sampling after informed consent. Three ml blood was collected and after centrifugation, serum was separated. Determination of ANA and anti dsDNA antibodies was performed by Enzyme Linked Immunosorbent Assay (ELISA). A $p \leq 0.05$ was considered as statistically significant.

Results: Overall mean age for group 1 was $29.66 \pm 8.07$ while CP patients had a mean age of $32.27 \pm 10.77$ which is slightly higher than group 1 . Only one individual in the CP group had ANA and dsDNA antibodies. While in the control group, two individuals had anti dsDNA and none of the subject had ANA.

Conclusion: Although it can be concluded from the findings of the present study that chronic periodontitis do not lead to production of auto antibodies, but studies on a larger sample size should be carried out to investigate this aspect.

Keywords: periodontitis, chronic periodontitis, auto antibodies, ELISA, ANA, dsDNA
Volume 9 Issue 5 - 2018

\author{
Aimen Batool,' Umaira Ghaffar,' Muhammad \\ Taimoor Iqbal, ${ }^{3}$ Sadia Minhas ${ }^{4}$ \\ 'Allied Hospital, Pakistan \\ ${ }^{2}$ Bakhtawar Amin Medical and Dental College, Pakistan \\ ${ }^{3}$ Nishtar Dental College, Pakistan \\ ${ }^{4}$ Department of Oral Pathology, Akhtar Saeed Medical and \\ Dental College, Pakistan
}

Correspondence: Aimen Batool,Allied Hospital, Faisalabad, Pakistan, Email draimenzaidi@gmail.com

Received: October 01, 2018 | Published: October 08, 2018

\section{Introduction}

The most frequent oral inflammatory conditions are periodontal diseases, which occur in reaction to bacterial plaque bio films, initiating damage to the alveolar bone, periodontal ligament and gingival (gums) exclusively all of which are types of supportive tissues of the teeth. Periodontitis arises mostly in $20 \%$ of adult populations, disturbing 300 million people globally. ${ }^{1}$

The periodontal disease is defined as a variety of inflammatory conditions. An inflammatory reaction at the gingival margin because of the increase of dental plaque is called as gingivitis. By keeping good oral hygiene, it is reversible. On the other hand, periodontitis is irreversible; which results in tissue damage and advanced loss of attachment and bone because of gingival epithelial relocation and this is further characterized by periodontal pocket formation and/or gingival recession. ${ }^{2}$

Now a day, researchers have a growing attention in studying the association among systemic health and periodontitis. Numerous studies have recommended a correlation among systemic autoimmune diseases, like rheumatoid arthritis and periodontitis. ${ }^{3-6}$

A study conducted in 2009 reported that in periodontal diseases autoimmune reactions are involved, wherever auto antigens is collagen type 1 is mainly evaluated. In addition to collagen type 1autoreactiveantibodies, numerous additional auto reactive antibodies have links with periodontal disease. Few of these containantiHSP60 antibodies (antibody related with the incidence and severity of coronary artery disease), anti-phospholipids (antibody related with blood clots and pregnancy difficulties such as stillbirth and miscarriage), anti desmosomal (antibody related with pemphigus) and anti-neutrophil cytoplasm (antibody related with systemic vasculitis). The pilot study established that in the periodontal disease progression autoimmunity is involved. ${ }^{?}$

In recent years a study conducted in Saudi Arabia, stated that no considerable association was seen among periodontal parameters and SLE (ANA, anti dsDNA) biomarkers. ${ }^{8}$ Though, few and unreliable data are seen among periodontitis and SLE association. Additionally, no study has evaluated if there is association among the autoimmune biomarkers in chronic periodontitis. This study was conducted to compare the frequency of auto antibodies in chronic periodontitis patients and systemically healthy controls

\section{Materials and methods}

This cross sectional study was carried out in the Department of Pathology, Allied Hospital Faisalabad, Pakistan. The study strictly followed the WMA Declaration of Helsinki and was approved by the Institutional Ethical Review Committee. Healthy individuals as controls and calculus associated CP patients from 18 to 55years were recruited from Dental Section, Allied Hospital Faisalabad. Pregnant females and patients with malignancy, autoimmunity, infectious disease, edentulous, xerostomia, or traumatic oral ulcers, having prosthesis or dentures, were excluded. Patients with periodontitis other than calculus associated $\mathrm{CP}$, for example, aggressive periodontitis, periodontitis associated with systemic diseases, and necrotizing periodontitis, were excluded. Periodontal examination of each subject was performed by the consultant dental surgeon. For the selected teeth, 
probing depth, clinical attachment loss, and bleeding on probing were recorded. CP patients with minimum ten natural teeth, attachment loss of $\geq 1 \mathrm{~mm}$ in $>30 \%$ of the sites examined, presence of abundant calculus, and radiographic confirmation of bone loss were included. Stages of CP were determined on the basis of criteria used by Wiebe and Putnins (2000). On the basis of clinical attachment loss (CAL), $\mathrm{CP}$ patients were subcategorized as mild (CAL 1-2mm) (10 patients), moderate (CAL 3-4mm) (16 patients), and severe (CAL $>5 \mathrm{~mm})(15$ patients). ${ }^{9}$ Blood samples of 90 subjects ( 45 with addictive habits and 45 without such habits) were taken by random sampling after informed consent. Three $\mathrm{ml}$ blood was collected and after centrifugation, serum was separated. Determination of ANA and anti dsDNA antibodies was performed by Enzyme Linked Immunosorbent Assay (ELISA). All the data were dealt with in an anonymous way. Statistical analysis SPSS version 20.0 was used for statistical calculations. $\mathrm{p} \leq 0.05$ was considered as statistically significant.

\section{Results}

Among 90 study subjects, group 1 comprised of 20 males and 25 females, whereas group 2 comprised of 24 males and 21 females. Overall mean age for group 1 was $29.66 \pm 8.07$ while CP patients had a mean age of $32.27 \pm 10.77$ which is slightly higher than group 1 .
When compared the gender distribution and mean age between these two groups, it was found to be insignificant statistically (Table 1). After obtaining general characteristics of both control and CP groups, detection of auto antibodies was carried out by ELISA technique. Only one individual in the CP group had ANA and dsDNA antibodies. While in the control group, two individuals had anti dsDNA and none of the subject had ANA. The associations between these two groups were analyzed for ANA and anti dsDNA, a negative association was observed in both groups. Table 2 shows the detailed values in two groups (Table 2).

Table I Number, percentage, mean $\pm S D$, and comparison of gender and age between two groups

\begin{tabular}{llll}
\hline Variables & $\begin{array}{l}\text { Healthy subjects } \\
(\mathbf{n = 4 5 )}\end{array}$ & $\begin{array}{l}\text { CP patients } \\
(\mathbf{n}=45)\end{array}$ & value \\
\hline Gender & & & \\
$M \quad n(\%)$ & $20(44.44 \%)$ & $24(53.33 \%)$ & 1.00 \\
F $\mathrm{n}(\%)$ & $25(43.56 \%)$ & $21(46.67 \%)$ & \\
Age $($ mean $\pm S D)$ & $29.66 \pm 8.07$ & $32.27 \pm 10.77$ & 0.07 \\
\hline
\end{tabular}

Statistically significant, CP, chronic periodontitis; $M$, male; F, female; $n$, number

Table 2 Comparison of auto antibodies between control and addictive habits groups

\begin{tabular}{llllll}
\hline & $\begin{array}{l}\text { Positive/negative } \\
\text { (Total controls = 45) }\end{array}$ & $\begin{array}{l}\text { Positive/negative (Total } \\
\text { addictive habits subjects=45) }\end{array}$ & Odds ratio & $\begin{array}{l}\mathbf{9 5 \%} \text { Cl } \\
\text { (Lower-upper) }\end{array}$ & P value \\
\hline ANA & $0 / 45$ & $1 / 45$ & 0.978 & $0.936-1.02$ & 0.5 \\
Anti dsDNA & $2 / 45$ & $1 / 45$ & 2.04 & $0.179-23.40$ & 1.000 \\
\hline
\end{tabular}

\section{Discussion}

Periodontitis is defined as a chronic inflammatory disorder of the periodontium which advances in reaction to local infections. Antigendriven host cell responses in periodontal disease act as start of chronic inflammatory disease; consequently, endogenous degenerative pathways mightreact as auxiliary magnification loops to local tissue destruction and proliferate inflammation. These routesmight be represented by the discharge of inflammatory cytokines, investigators tried to relate numerous pathological and clinical objects to the detailed immune responses produced ${ }^{10}$ Many studies reported that as compared to females, male population has advanced periodontal destruction. The etiology behind this is not clear, but it is proposed that it might be due to poor oral hygiene which is most frequently seen in males. Though the association among the disease and gender is not clear and is considered as reliable factor. Therefore, gender might be a demographic factor, which might restrict the results of other factors and for investigation the disease it must be controlled. ${ }^{11-14}$ Brandtzaeg and Kraus (1965) were the first to suggest the role of autoimmunity in the etiology of periodontal diseases. The role of autoimmunity in periodontal disease has been reported since last $30 y e a r s$. In the past decades lots of studies reported to support the idea of autoimmunity role in periodontal diseases. ${ }^{15}$ It is proposed that periodontal disease include strong autoimmune component, which uninterruptedlyinspiresattention in discovering the association among autoimmune diseases and periodontal. Studies conducted on the effects of SLE on periodontitis are inadequate and questionable. There are case reports on cutaneousand systemic SLE patients displaying aggressive periodontitis in these patients. ${ }^{16-18}$ Other studies described occurrence of ANA in, Japanese as 9.5\%, Indians it was
$12.3 \%$, whereas for US population at $13.8 \% .^{19-21}$ The consider ably increased incidence of ANA in these studies may be because of larger study populations, environmental factors, genetic factor sand most significantly, gender associated result sand similarly in the present study only male population were included. This assessment of ANA between four different states expresses the source for the belief that ANA occurrence between adults could differ by geographic demographic and geographic factors.

A study supported out by Azizah et al., ${ }^{21}$ described that in general public of Malaysia, ANA occurrence at $5.5 \%$, whereas $0 \%$ prevalence of anti dsDNA antibodies. ${ }^{22}$ A study conducted in Northern Sweden, stated that numerous years before the appearance of clinical signs and symptoms, auto antibodies can be noticed in the serum of persons who progress to systemic lupus erythematosus. ${ }^{23}$ In conclusion, there were no alterations in periodontal parameters among patients with SLE and normal healthy individuals. As well as, no noteworthy association was found among SLE biomarkers and periodontal parameters. This similarity might be due to anti-inflammatory medication taken by SLE patients, and/or possible lack of genetic variation. Within SLE patients, those who suffer from arthritis had more periodontal tissue destruction compared with those without arthritis. Further studies are needed to verify the results of this study. ${ }^{24}$

\section{Conclusion}

Although it can be concluded from the findings of the present study that chronic periodontitis is not associated with production of auto antibodies/autoimmunity, but studies on a larger sample size should be carried out to investigate this aspect. 


\section{Acknowledgements}

None.

\section{Conflict of interest}

The author declares no conflict of interest.

\section{References}

1. Petersen PE, Baehni PC. Periodontal health and global public health. Periodontol. 2012;60(1):7-14.

2. Anonymous. American academy of periodontology task force report on the update to the 1999 classification of periodontal diseases and conditions. J Periodontol. 2015;86(7):835-838

3. Al- Katma MK, Bissada NF, Bordeaux JM, et al. Control of periodontal infection reduces the severity of active rheumatoid arthritis. J Clin Rheumatol. 2007;13(3):134-137.

4. Ortiz P, Bissada NF, Palomo L, et al. Periodontal therapy reduces the severity of active rheumatoid arthritis in patients treated with or without tumor necrosis factor inhibitors. J Periodontol. 2009;80(4):535-540.

5. Scher JU, Bretz WA, Abramson SB. Periodontal disease and subgingival microbiota as contributors for rheumatoid arthritis pathogenesis: modifiable risk factors? Curr Opin Rheumatol. 2014;26(4):424-429.

6. Fabbri C, Fuller R, Bonfa E, et al. Periodontitis treatment improves systemic lupus erythematosus response to immunosuppressive therapy. Clin Rheumatol. 2014;33(4):505-509.

7. Koutouzis T, Haber D, Shaddox L, et al. Autoreactivity of Serum Immunoglobulin to Periodontal Tissue Components: A Pilot Study. J Periodontol. 2009;80(4):625-633.

8. Al- Mutairi KD, Al- Zahrani MS, Bahlas SM, et al. Periodontal findings in systemic lupus erythematosus patients and healthy controls. Saudi Medical Journal. 2015;36(4):463-468.

9. Wiebe C, Putnins E. "The periodontal disease classification system of the American Academy of Periodontology- an update." Can Dent Assoc. 2000;66(11):594-597.

10. Dongari- Bagtzoglou I, Ebersole JL. "Increased presence of interleukin6 (IL-6) and IL- 8 secreting fibroblast subpopulations in adult periodontitis." Journal of Periodontology. 1998;69(8):899-910.

11. Grossi SG, Genco RJ, Machtei EE, et al. Assessment of risk for periodontal disease. II. Risk indicators for alveolar bone loss. Journal of Periodontology. 1995;66(1):23-29.
12. Meisel P, Reifenberge J, Haase R, et al. "Women are periodontally healthier than men, but why don't they have more teeth than men?" Menopause. 2008;15(2):270-275.

13. Mundt T, Schwahn C, Mack F, et al., "Risk indicators for missing teeth in working-age pomeranians - an evaluation of high-risk populations." Journal of Public Health Dentistry. 2007;67(4):243-249.

14. Slade GD, Spence AJ. Periodontal attachment loss among adults aged $60+$ in South Australia. Community Dentistry and Oral Epidemiology. 1995;23(4):237-242.

15. Koutouzis T, Haber D, Shaddox L, et al. Autoreactivity of serum immunoglobulin to periodontal tissue components: a pilot study. J Periodontol. 2009;80(4):625-633.

16. Nair S, Faizuddin M, Dharmapalan J. Role of autoimmune responses in periodontal disease. Autoimmune Dis. 2014;2014:596824.

17. Nagler RM, Lorber M, Ben-Arieh Y, et al. Generalized periodontal involvement in a young patient with systemic lupus erythematosus. Lupus. 1999;8(9):770-772.

18. Tietmann C, Bissada NF. Aggressive periodontitis in a patient with chronic cutaneous lupus erythematosus: a case report. Quintessence Int. 2006;37(5):401-408

19. Satoh M, Chan EK, Ho LA, et al. Prevalence and sociodemographic correlates of antinuclear antibodies in the United States. Arthritis Rheum. 2012;64(7):2319-2327.

20. Hayashi N, Koshiba M, Nishimura K, et al. Prevalence of disease- specific antinuclear antibodies in general population: estimates from annual physical examinations of residents of a small town over a 5-year period. Mod Rheumatol. 2008;18(2):153-60.

21. Minz RW, Kumar Y, Anand S, et al. Antinuclear antibody positive autoimmune disorders in North India: an appraisal. Rheumatol Int. 2012;32(9):2883-8.

22. Azizah MR, Azila MN, Zulkifli MN, et al. The prevalence of antinuclear, anti- dsDNA, anti- Sm and anti- RNP antibodies in a group of healthy blood donors. Asian Pac J Allergy and Immunol. 1996;14(2):125.

23. Eriksson $\mathrm{C}$, Kokkonen $\mathrm{H}$, Johansson M, et al. Auto antibodies predate the onset of systemic lupus erythematosus in northern Sweden. Arthritis Res \& Ther. 2011;13(1):R30.

24. Al- Mutairi, Khalid D. et al. Periodontal findings in systemic lupus erythematosus patients and healthy controls. Saudi Medical Journal. 2015;36(4):463-468. 